\title{
High-Dose Cytarabine-Mitoxantrone Versus Hyper-CVAD in Adult Acute Lymphoblastic Leukemia and Burkitt's Lymphoma: A Single Center Experience of Two Induction Regimens
}

\author{
Quanyi $\mathrm{Lu}^{1,2}$, Woondong Jeong ${ }^{1}$, Sreedhar Katragadda ${ }^{1}$, Karen Seiter ${ }^{1}$, Qiuhu $\mathrm{Shi}^{3}$ and \\ Delong Liu ${ }^{*}, 1$
}

\author{
${ }^{I}$ Division of Oncology and Hematology, New York Medical College and Westchester Medical Center, Munger Pavilion \\ 250, Valhalla, NY 10595, USA \\ ${ }^{2}$ Xiamen Zhongshan Hospital, Xiamen, Fujian Province, China \\ ${ }^{3}$ Department of Biostatistics, New York Medical College Vosburg Pavilion, Valhalla, NY 10595, USA
}

\begin{abstract}
The treatment of acute lymphoblastic leukemia (ALL) in children has made significant progress. However, the treatment for adult ALL patients has been less successful. The majority of adult patients develop recurrent disease and subsequently die of their leukemia. This study reports a single center experience of adult ALL therapy with two different induction regimens. 73 adult patients with newly diagnosed ALL were treated at the Westchester Medical Center. These patients received induction chemotherapy with either high dose mitoxantrone and high-dose cytarabine (HDAM, n=52) or Hyper-CVAD $(n=21)$. The complete remission $(C R)$ rate was $87 \%$ in the HDAM group and $76 \%$ in the Hyper-CVAD group ( $\mathrm{p}=0.31)$. The median CR duration was 34 months $(95 \% \mathrm{CI}, 14-)$ for the HDAM group, and 18 months (95\% CI, 9 -) for the hyper-CVAD group, respectively. The median overall survival (OS) for patients in the HDAM group was 21 months (95\% confidence interval [CI], 13 - 35 months). The 3-year and 5-year OS was 35\% and 30\%, respectively. In the Hyper-CVAD group, median OS was 27 months (95\% CI, 12 -), with a 3-year OS of 44\%. The difference of CR duration and OS between the two groups was not statistically significant ( $\mathrm{p}=0.86$ for $\mathrm{CR}, \mathrm{p}=0.73$ for OS). The statistically significant favorable prognostic factors for overall survival include HDAM induction, karyotyping other than $\mathrm{t}(9 ; 22)$ and $\mathrm{t}(4 ; 11)$, day 1 platelet count $\geq 20,000 \times 10^{6} / \mathrm{L}$, age $<35$, day $1 \mathrm{WBC}<10 \times 10^{6} / \mathrm{L}$. In conclusion, the two regimens are comparable in this retrospective analysis for ALL induction from a single center. HDAM induction was found to be a favorable prognostic factor for overall survival.
\end{abstract}

Keywords: Mitoxantrone, hyper-CVAD, acute lymphoblastic leukemia, Burkitt's lymphoma.

\section{INTRODUCTION}

The treatment of acute lymphoblastic leukemia and Burkitt's lymphoma (ALL) in children has made significant progress. The complete remission (CR) rate exceeds $90 \%$ and the long-term survival rate approaches $80 \%[1,2]$. However, the treatment for adult ALL patients has been far less successful. Although CR rates in newly diagnosed ALL patients are now $>80 \%$, the duration of remission is still short. The majority of adult patients develop recurrent disease and subsequently die of their leukemia [3-6]. This is especially true for elderly patients, whose median survival is less than 1 year [7].

Recent results of clinical trials have shown that intensive chemotherapy regimens can improve the outcome for patients with adult ALL [8]. With these regimens, the longterm survival rates range from $30 \%$ to $45 \%$ [5, 8-12]. These regimens usually consist of four or five drugs (vincristine, prednisone, anthracycline, cyclophosphamide, and asparaginase). The results of these treatment programs have been

*Address correspondence to this author at the Division of Oncology and Hematology, New York Medical College and Westchester Medical Center, Munger Pavilion 250, Valhalla, NY 10595, USA; Tel: (914)493-7514; Fax: (914)594-4420; E-mail: delong_liu@nymc.edu comparable, and no single regimen was proven to be superior to others [12-14].

Arlin et al. developed a dose-intensive chemotherapy regimen for ALL using high dose mitoxantrone combined with high dose Ara-C (HDAM) [15]. The results of treatment were encouraging and toxicity was acceptable. Using a similar regimen, Weiss et al. treated 37 patients with newly diagnosed adult ALL. Thirty- one patients (84\%) achieved CR. The median time to $\mathrm{CR}$ was 34 days and the toxicity was acceptable [16]. These results showed that high dose chemotherapy with Ara-C /Mitoxantrone appears superior to traditional four-drug induction in terms of $\mathrm{CR}$, failure with resistant disease, and activity in $\mathrm{Ph}+$ disease.

Kantarjian et al. have reported favorable results using dose-intensive Hyper-CVAD (fractionated cyclophosphamide, vincristine, doxorubicin, and dexamethasone alternating with high-dose methotrexate and Ara-C) regimen in ALL. The CR rate was $91 \%$ among 185 patients, and the 5year survival rates were $39 \%[17,18]$. The results of HyperCVAD therapy seems to be superior to other previous regimens in historical controls [5, 9-11, 18]. It is however uncertain whether intensification of induction therapy can lead to improvement of long term survival due to the lack of direct comparison of different regimens. So far there is no study 
directly comparing HDAM and Hyper-CVAD. We report a retrospective analysis of 73 adult ALL patients treated with either Hyper-CVAD or HDAM in a single institution.

\section{PATIENTS AND METHODS}

\section{Patients}

Between January 1994 and January 2005, 73 previously untreated consecutive adult patients (age $>18$ years) had a diagnosis of ALL or lymphoblastic lymphoma. These patients received either high dose mitoxantrone and high-dose AraC (cytarabine) (HDAM) or Hyper-CVAD. Informed consent was obtained according to institutional guidelines. The induction regimen was chosen per investigators' preferences and was not based on risk stratification. However, patients with lymphoblastic lymphoma were predominantly treated with Hyper-CVAD.

\section{Induction, Consolidation and Maintenance Therapy}

The HDAM induction regimen consists of Ara-C $3 \mathrm{gm}$ $/ \mathrm{m}^{2}$ /day by 3 -hour intravenous (IV) infusion for 5 days plus mitoxantrone $80 \mathrm{mg} / \mathrm{m}^{2}$ IV given as a single dose on day 2 or day 3. This was reported previously $[15,16]$. Intrathecal methotrexate dose was $6 \mathrm{mg} / \mathrm{m}^{2}$ (maximal dose $15 \mathrm{mg}$ ) given on days 2 and 4.

The consolidation schedule was as reported earlier [16] with slight modifications as the following: Consolidation A: 7-14 days after hospital discharge, the patient was given consolidation A; Vincristine $2 \mathrm{mg} / \mathrm{m}^{2}$ IV given on days $1,8,15$, 22 and 29. The maximum dose for patients $<60$ years was 4 $\mathrm{mg}$. The maximum dose for patients $>60$ years was $2.5 \mathrm{mg}$. Subsequent doses were reduced by $50 \%$ for Grade 3 neurotoxicity and omitted for Grade 4 toxicity. Prednisone 60 $\mathrm{mg} / \mathrm{m}^{2} /$ day was given orally in two divided doses. The prednisone was given for days 1 through 30 during consolidation A. Following day 30 the prednisone was tapered over approximately 10 days. While on prednisone, patients received gastrointestinal prophylaxis. Intrathecal methotrexate 6 $\mathrm{mg} / \mathrm{m}^{2}$ (max $15 \mathrm{mg}$ ) was given 4 times during consolidation A (e.g. on days 8, 15, 22 and 29). Intrathecal therapy could be deferred for patients with circulating blasts, severe thrombocytopenia, or coagulopathy. Patients who could not tolerate (or whose CNS disease was resistant to) methotrexate received Ara-C $30 \mathrm{mg} / \mathrm{m}^{2}$. Sulfamethoxizole /trimethoprim one double-strengh tablet PO BID was given 3 days each week while on full dose prednisone (days 1-30). During day 31-47 of prednisone tapering, Sulfamethoxizole /trimethoprim was given daily BID. Patients unable to take sulfamethoxizole/trimethopirm prophylaxis received aerosolized or IV pentamidine every 14 days. During consolidation A (typically approximately 60-70 days following initiation of induction therapy), vincristine was held if clinically indicated for $\mathrm{ANC}<1,000 / \mathrm{ul}$ and platelets $<100,000 / \mathrm{ul}$. Response status was determined following the end of consolidation A. Patients in complete remission went on to consolidation B 2 weeks after the last dose of vincristine.

Consolidation B: Cyclophosphamide $4 \mathrm{~g} / \mathrm{m}^{2}$ IV for one single dose. Patients older than 60 years of age received $3 \mathrm{~g} / \mathrm{m}^{2}$.

Consolidation C: Approximately 3-4 weeks after receiving cyclophosphamide, patients were admitted to the hospital for placement of Ommaya reservoir. After recovering from surgery (usually 2-4 days) patients began consolidation C; Etoposide $200 \mathrm{mg} / \mathrm{m}^{2} / \mathrm{d}$ IV over three hours given daily from day 1 to day 3 . Ara-C $25 \mathrm{mg} / \mathrm{m}^{2}$ IV bolus followed by 200 $\mathrm{mg} / \mathrm{m}^{2} / \mathrm{d}$ IV continuous infusion $\mathrm{x} 4$ days. Intrathecal methotrexate $6 \mathrm{mg} / \mathrm{m}^{2}$ (max $15 \mathrm{mg}$ ) was given twice during consolidation $\mathrm{C}$.

Consolidation D: L-asparaginase was given at a dose of $10,000 \mathrm{I} . \mathrm{U} / \mathrm{m}^{2}$ IM or IV TIW x 6 doses. The dose for patients $>60$ years was 6,000 I.U/m 2 .

\section{Maintenance}

At the completion of consolidation and upon recovery of normal blood counts, patients in remission received two years of maintenance. Maintenance consisted of repeating alternating sequences of chemotherapy. For patients who received fewer than 5 cycles of maintenance chemotherapy during this 2 year period due to treatment delays for blood count recovery or other complications, the maintenance phase was extended beyond 2 years to complete a full $5 \mathrm{cy}-$ cles of maintenance therapy.

Sequence 1: Vincristine $2 \mathrm{mg} / \mathrm{m}^{2}$ i.v. given on days 1 and 8 (max $4 \mathrm{mg}$ ). Patients greater than 60 years of age received $1 \mathrm{mg} / \mathrm{m}^{2}$ (max $2 \mathrm{mg}$ ). Prednisone $50 \mathrm{mg} / \mathrm{m}^{2} /$ day (which can be rounded to the nearest $20 \mathrm{mg}$ ) given orally in two divided doses days 1-8. The prednisone was stopped without taper on day 8 . Doxorubicin $60 \mathrm{mg} / \mathrm{m}^{2}$ i.v. given on day 15 . Left ventricular function was evaluated prior to Doxorubicin. For patients who experienced a greater than or equal to $10 \%$ deterioration in LV function (or an LVEF $<45 \%$ ), Carmustine /Cyclophosphamide was substituted for doxorubicin. This was followed with 6-Mercaptopurine (6-MP) $90 \mathrm{mg} / \mathrm{m}^{2} / \mathrm{d}$ (rounded to the nearest $50 \mathrm{mg}$ ) given orally in two or three divided doses on days 36-64. 6-MP was discontinued if WBC and platelet count fell below 1,500/ul and 100,000/ul, respectively. Methotrexate $15 \mathrm{mg} / \mathrm{m}^{2} / \mathrm{d}$ (maximum dose 25 $\mathrm{mg}$ ) given orally days $39,46,53$, and 60 . Methotrexate was discontinued if $\mathrm{WBC}$ and platelet count fell below 1,500/ul and $100,000 / \mathrm{ul}$ respectively. Methotrex-ate was withheld for serum creatinine $>2.0 \mathrm{mg} / \mathrm{dl}$. Intrathecal methotrexate 6 $\mathrm{mg} / \mathrm{m}^{2}$ (maximum dose $15 \mathrm{mg}$ ) was given twice during each sequence of maintenance (between days 36-64). A dose of dactinomycin $1 \mathrm{mg} / \mathrm{m}^{2}$ i.v. was given on day 85 . This was held if clinically indicated for ANC $<1,000 / \mathrm{ul}$ and platelets $<100,000 \mathrm{ul}$. The next sequence of maintenance started in 14 days.

Sequence 2: Identical to the first sequence except that doxorubicin (day 15) was replaced by carmustine (BCNU) and cyclophosphamide. Carmustine (BCNU) $80 \mathrm{mg} / \mathrm{m}^{2}$ i.v. with cyclophosphamide $800 \mathrm{mg} / \mathrm{m}^{2}$ i.v. were given on day 15 .

Hyper-CVAD induction regimen consisted of two phases: a dose-intensive phase with four cycles of HyperCVAD alternating with four cycles of high-dose MTX and Ara-C and a maintenance phase with POMP as reported previously [17]. Patients who had $\mathrm{Ph}+\mathrm{ALL}$ received imatinib since it became available. Patients who had initial WBC $\geq$ $100,000 \times 10^{6} / \mathrm{L}$ at presentation underwent leukapheresis.

All patients received prophylactic antibiotics: ciprofloxacin $500 \mathrm{mg}$ PO BID, acyclovir $400 \mathrm{mg}$ PO BID or valacyclovir 500mg PO QD, itriconazole $200 \mathrm{mg}$ PO BID or voriconazole 200mg PO BID. 


\section{Response Criteria}

CR was defined as disappearance of all clinical evidence of leukemia for a minimum of 4 weeks demonstrating normal bone marrow cellularity with normal granulopoiesis, thrombopoiesis, and less than 5\% blasts. Requirements for peripheral-blood counts included neutrophil count greater than 1,000 x $10^{6} / \mathrm{L}$, platelet count greater than $100,000 \times 10^{6} / \mathrm{L}$, and no circulating blasts. Treatment failure or resistant disease is defined as not achieving a CR by repeating bone marrow biopsy at the recovery of peripheral $\mathrm{WBC}$ or at day 45 . CR duration was calculated from the date of achievement of CR until evidence of leukemia recurrence. Overall survival was measured from the start of treatment until death or last follow-up. Patients undergoing allogeneic stem cell transplant were censored in $\mathrm{CR}$ for remission duration.

\section{Statistical Methods}

Survival and CR duration were estimated using the Kaplan-Meiyer method and compared using the Log-rank test. Differences in CR rates by treatment and by prognostic factors were analyzed using a $\chi^{2}$ test and Fisher's exact test.

\section{RESULTS}

\section{Patient Characteristics}

The characteristics of the 73 patients enrolled are summarized in Table 1. They are divided into two groups, HDAM and CVAD.

\section{Table 1. Patient Characteristics}

\begin{tabular}{|c|c|c|}
\hline & HDAM & CVAD \\
\hline \hline No. & 52 & 21 \\
\hline Age, median (range) & $35(19-78)$ & $29(19-68)$ \\
\hline Age $<35$ & $25(48 \%)$ & $12(57 \%)$ \\
\hline$\geq 35$ & $27(52 \%)$ & $9(43 \%)$ \\
\hline Men & $32(62 \%)$ & $11(52 \%)$ \\
\hline WBC, median (range) $\times 10^{6} / \mathrm{L}$ & $8(0.1-115)$ & $14(1.4-660)$ \\
\hline$*$ WBC $\leq 10,000 \times 10^{6} / \mathrm{L}$ & $31(63 \%)$ & $9(43 \%)$ \\
\hline$>10,000 \times 10^{6} / \mathrm{L}$ & $18(37 \%)$ & $12(57 \%)$ \\
\hline${ }^{*}$ PLT $<20,000 \times 10^{6} / \mathrm{L}$ & $12(25 \%)$ & $6(29 \%)$ \\
\hline$\geq 20,000 \times 10^{6} / \mathrm{L}$ & $37(75 \%)$ & $15(71 \%)$ \\
\hline${ }^{*} \mathrm{LDH}<600 \mathrm{U} / \mathrm{L}$ & $20(43 \%)$ & $9(43 \%)$ \\
\hline$\geq 600 \mathrm{U} / \mathrm{L}$ & $26(57 \%)$ & $12(57 \%)$ \\
\hline Cytogenetics t(9;22) and t(4;11) & $8(15 \%)$ & $2(10 \%)$ \\
\hline $\mathrm{t}(8 ; 14)$ & $0(0 \%)$ & $2(10 \%)$ \\
\hline others & $44(85 \%)$ & $17(80 \%)$ \\
\hline Histology Subtype Pre-B & $45(86 \%)$ & $11(52 \%)$ \\
\hline Burkitt & $0(0 \%)$ & $3(14 \%)$ \\
\hline $\mathrm{T}$ & $7(14 \%)$ & $7(34 \%)$ \\
\hline
\end{tabular}

* Some patients were transferred from other hospitals, and values of initial lab tests were not available. This includes 3 patients without initial WBC and platelets and 6 patients without initial LDH in HDAM group.
Fifty-two patients ( 20 women and 32 men) with a median age of 35 years (range 19 to 78 ) were treated with HDAM regimen, 21 patients (10 women and 11 men) with a median age of 29 years (range 19 to 68) were treated with hyperCVAD regimen. Six patients $(14 \%)$ had $t(9 ; 22)$ Philadelphia chromosome, and $2(5 \%)$ had $\mathrm{t}(4 ; 11)$ in HDAM. In HyperCVAD group, one patient had $\mathrm{t}(4 ; 11)$, one other was $\mathrm{Ph}+$. In general, the groups are similar in respect to the incidence of adverse prognostic features with the exception that HyperCVAD group had more patients with Burkitt's lymphoma.

\section{Treatment Results}

In the HDAM group, the median follow-up was 52 months (range, 0.5 - 119 months, 95\% CI 41-106). The median follow-up for the Hyper-CVAD group was 25 months (range, 0.5 - 53 months, 95\% CI 22-35). Median follow-up of all 73 patients combined was 41 months (range, 0.5 - 119 months, 95\% CI $30-53$ ).

Forty-five (87\%) of 52 patients in the HDAM group achieved a complete remission (CR), 2 (4\%) had resistant disease and $5(9 \%)$ died during remission induction. The primary causes of death were bacterial and /or fungal infection.. In Hyper-CVAD group, $16(76 \%)$ of 21 patients achieved CR, 4 (19\%) had resistant disease and 1 (5\%) died during induction. The cause of death was also serious infection. The CR rate was not statistically different between the two groups $(\mathrm{p}=0.31)$.

The median overall survival (OS) for patients in the HDAM group was 21 months (95\% confidence interval [CI], 13 - 35 months). The 3-year and 5-year OS was 35\% and $30 \%$, respectively (Fig. 1). In the Hyper-CVAD group, median OS was 27 months (95\% CI, 12 -), with a 3-year OS of $44 \%$. The median CR duration was 34 months (95\% CI, 14-) for the HDAM group, and 18 months $(95 \% \mathrm{CI}, 9$-) for the hyper-CVAD group, respectively. The difference of CR duration and OS between the two groups was not statistically significant $(\mathrm{p}=0.86$ for $\mathrm{CR}, \mathrm{p}=0.73$ for $\mathrm{OS})$.

Due to the limited number of patients, the two groups of patients were combined for analysis of prognostic factors. The chromosome abnormalities of $t(9 ; 22)$ and $t(4 ; 11)$ were poor-risk factors. OS was significantly better $(p=0.04)$ for those in favorable group $v s$ poor-risk group (median survival 29 months without poor-risk factors vs 13 months with poorrisk factors). Other prognostic factors [including sex, age, histology (Pre-B, Burkitt's, T), day 1 WBC count $(\leq 10,000$ or $\left.>10,000 \times 10^{6} / \mathrm{L}\right)$, day 1 platelet $\operatorname{count}(<20,000$ or $\left.\geq 20,000 \times 10^{6} / \mathrm{L}\right)$, day $\left.1 \mathrm{LDH}(<600,>600 \mathrm{U} / \mathrm{L})\right]$ were not statistically significant. CR duration was significantly better in $\mathrm{T}$ cell vs pre- B histology $(\mathrm{p}=0.048)$ (median CR duration; 50 months vs 16 months).

For overall survival, the following were found to be statistically significant favorable prognostic factors: HDAM induction, karyotyping other than $\mathrm{t}(9 ; 22)$ and $\mathrm{t}(4 ; 11)$, Day 1 platelet count $\geq 20,000$, Age $<35$, Day $1 \mathrm{WBC}<10$.

\section{Toxicity}

Induction therapy with either HDAM or hyper-CVAD regimen caused universal myelosuppression. The induction mortality for HDAM was $9 \%$, and $5 \%$ for Hyper-CVAD, 


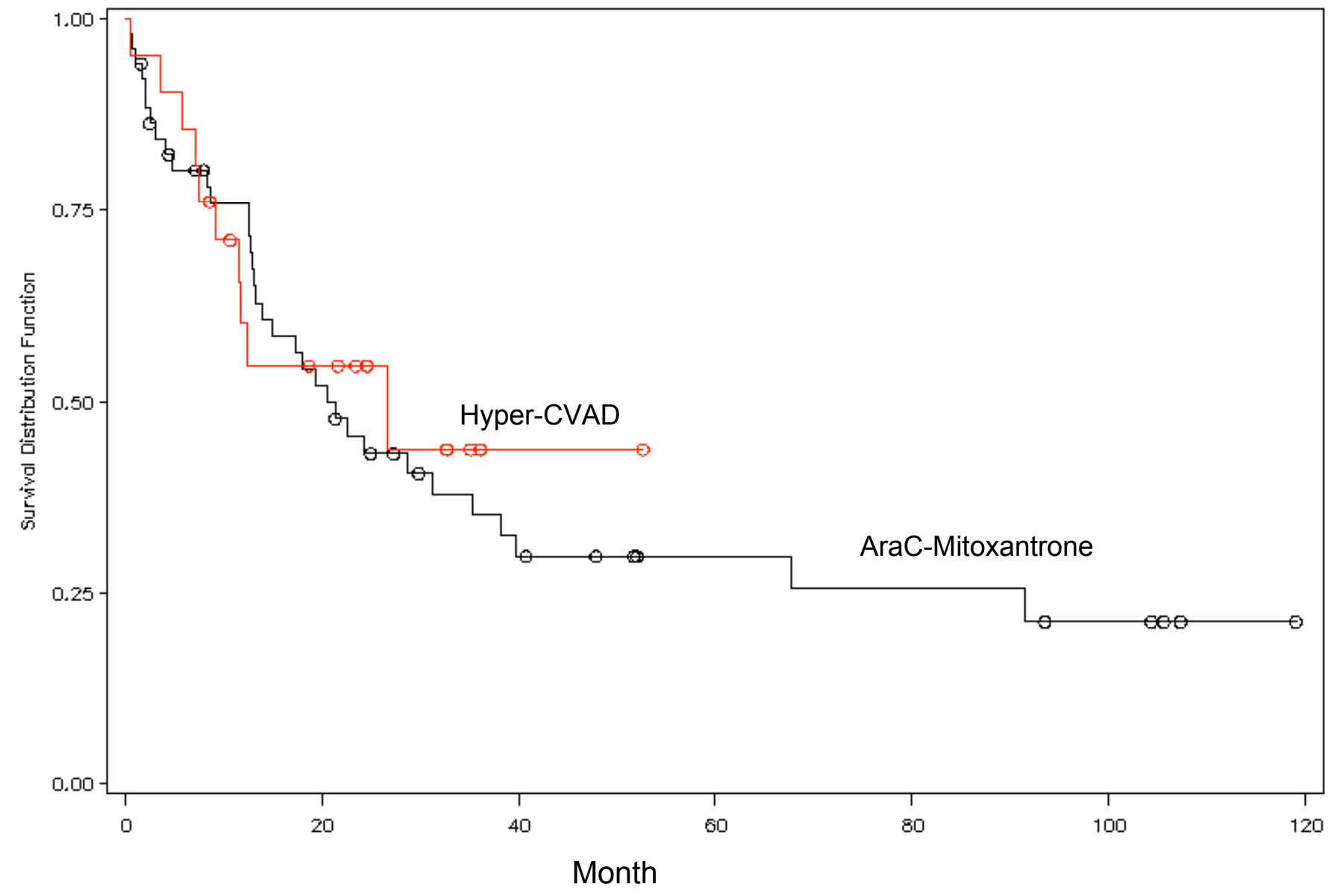

Fig. (1). Overall survival of ALL patients after induction with high-dose cytarabine plus mitoxantrone (HDAM) and with hyper-CVAD (CVAD). The difference of OS between the two groups was not statistically significant $(\mathrm{p}=0.73)$.

which are not statistically significant. The predominant nonhematologic toxicity was neutropenic fever and infection requiring intravenous antibacterials and antifungals in almost all patients. Hepatic and cardiac toxicity were not significantly different between the two groups. Fungal infection manifested as parasinusitis, fungal pneumonia, and fungemia. The most common cause of death during induction was septic shock with bacterial and/or fungal infection. Other rare contributing causes of death were ARDS with leukemic pulmonary infiltration. There was 1 death from necrotizing pancreatitis after L-asparaginase injection.

\section{DISCUSSION}

There have been few prospective randomized clinical studies of adult ALL induction therapy in recent years. This study reported a single center experience of two different induction regimens initially developed from two large cancer centers. The CR rate was $87 \%$ in HDAM group and $76 \%$ in Hyper-CVAD group $(p=0.31)$. These results appear to be similar to those reported from previous studies $[5,19,20]$. In this single center retrospective analysis, there are no significant differences in $\mathrm{CR}$ rate, median survival and induction mortality between the two groups (Table 2). Although it is difficult to compare directly with historical studies, they nevertheless appear to be similar to those reported in the literature (Table 3). Mortality during induction typically ranged between $5 \%$ to $10 \%[1,2]$. Multivariate analysis has demonstrated that WBC count at presentation, age, immunophenotype, Philadelphia chromosome-positive $(\mathrm{Ph}+)$ disease, and time to $\mathrm{CR}$ are important prognostic factors for adult ALL. This is consistent with the previous reports [16, 21$23]$. Other than karyotyping, age is the most important prognostic factor. The shorter time to $\mathrm{CR}$ is associated with longer survival. Patients achieving early CR are more likely to be long-term survivors $[22,23]$. One of the major purposes for using short course, dose-intensive chemotherapy for ALL is for rapid reduction of the leukemic burden to minimize the development of drug resistance. Both HyperCVAD and HDAM produced early CR (29 days vs 27 days).

Intensive chemotherapy regimen containing high dose Ara-C has led to better responses for mature B-ALL, ProBALL and relapsed or refractory adult ALL as well as overt CNS leukemia [21, 24, 25]. Recent study has shown that Hyper- CVAD improved the CR rate in Ph+ ALL [26, 27].

Hyper- CVAD regimen has been used to treat mantle cell lymphoma [28], multiple myeloma [29], and lymphoblastic lymphoma [26, 27]. Rituximab and imatinib have been incorporated into this regimen for the therapy of CD20- positive ALL and Ph-positive ALL, respectively [26, 27, 30]. Clofarabine (Compound 506) has recently been approved for the therapy of T-ALL [31]. Further efforts are also being made to test new combinations to improve on drug pharmacokinetics [32]. From this study we found that HDAM in- 
duction is a favorable prognostic factor for overall survival. We have therefore initiated a clinical trial to use HDAM regimen for induction and intensify the consolidation with Hyper-CVAD regimen.

Table 2. Comparison of HDAM with Hyper-CVAD on Treatment Outcome

\begin{tabular}{|l|c|c|}
\hline & HDAM (n) & CVAD (n) \\
\hline \hline No & 52 & 21 \\
\hline Incidence of CR & $87 \%(45)$ & $76 \%(16) \mathrm{p}=0.31$ \\
\hline Failure with resistant disease & $4 \%(2)$ & $19 \%(4)$ \\
\hline Deaths during induction & $9 \%(5)$ & $5 \%(1)$ \\
\hline Time to CR (median days) & 29 & 27 \\
\hline CR duration (median months) & 34 & $18 \mathrm{p}=0.86$ \\
\hline Median survival (months) & 21 & $27 \mathrm{p}=0.73$ \\
\hline 3yr OS & $35 \%$ & $44 \%$ \\
\hline 5yr OS & $30 \%$ & $\mathrm{NR}$ \\
\hline
\end{tabular}

HDAM: high-dose AraC and Mitoxantrone; NR; not reached.

Table 3. Comparison of Clinical Studies on HDAM and Hyper-CVAD

\begin{tabular}{|l|c|c|c|c|}
\hline & $\begin{array}{c}\text { MSKCC } \\
\text { HDAM }\end{array}$ & $\begin{array}{c}\text { MDACC } \\
\text { CVAD }\end{array}$ & $\begin{array}{c}\text { NYMC } \\
\text { HDAM }\end{array}$ & $\begin{array}{c}\text { NYMC } \\
\text { CVAD }\end{array}$ \\
\hline \hline Patient No. & 37 & 204 & 52 & 21 \\
\hline Median age (range) & $39(18-72)$ & $39.5(16-79)$ & $35(19-78)$ & $29(19-68)$ \\
\hline Men & $60 \%$ & $65 \%$ & $62 \%$ & $52 \%$ \\
\hline Ph+ & $17 \%$ & $16 \%$ & $9 \%$ & $5 \%$ \\
\hline WBC & 28 & 7.7 & 8 & 14 \\
\hline B & $71 \%$ & $76 \%$ & $86 \%$ & $52 \%$ \\
\hline T & $28 \%$ & $18 \%$ & $14 \%$ & $34 \%$ \\
\hline AlloSCT & $9 \%$ & NA & $8 \%$ & $14 \%$ \\
\hline CR & $83 \%$ & $91 \%$ & $87 \%$ & $76 \%$ \\
\hline Induction Death & $9 \%$ & $6 \%$ & $9 \%$ & $5 \%$ \\
\hline Resistant disease & $8 \%$ & $3 \%$ & $4 \%$ & $19 \%$ \\
\hline Time to CR(median) & 32 & 21 & 29 & 27 \\
\hline $5 y r$ OS & $35 \%$ & $39 \%$ & $30 \%$ & NA \\
\hline Median OS(months) & 24 & 35 & 21 & 27 \\
\hline CR in Ph+ (No) & $85 \%$ & $91 \%$ & $100 \%(6)$ & $100 \%(1)$ \\
\hline $5 y r$ OS in Ph+ & $26 \%$ & $7 \%$ & NA & NA \\
\hline
\end{tabular}

MSKCC: Memorial Sloan-Kettering Cancer Center; MDACC: MD Anderson Cancer Center; HDAM: high-dose AraC and Mitoxantrone; CVAD: Hyper-CVAD; NYMC: New York Medical College; NA:not available.

\section{ACKNOWLEDGEMENT}

Quanyi $\mathrm{Lu}$ and Woondong Jeong contributed equally to this study. This work is supported in part by a grant from New York Medical College Blood Diseases Fund (DL) and by a fellowship grant from Xiamen Zhongshan Hospital, China (QL).

\section{REFERENCES}

[1] Pui CH, Relling MV, Sandlund JT, Downing JR, Campana D, Evans WE. Rationale and design of total therapy study XV for newly diagnosed childhood acute lymphoblastic leukemia. Ann Hematol 2004; 83: S124-S6.

[2] Pui C-H, Evans WE. Treatment of acute lymphoblastic leukemia. N Engl J Med 2006; 354: 166-81.

[3] Annino L, Vegna ML, Camera A, et al. Treatment of adults acute lymphoblastic leukemia(ALL) long term follow-up of the GIMEMA ALL0288 randomized study. Blood 2002; 99: 863-71.

[4] Linker CA, Levitt LJ, O'Donnell M, et al. Treatment of adult acute lymphoblastic leukemia with intensive cyclical chemotherapy: a follow-up report. Blood 1991; 78: 2814-22.

[5] Kantarjian HM, Walters RS, Keating MJ, et al. Results of the vincristine, Doxorubicin and dexamethasone regimen in adult with acute lymphocytic leukemia. J Clin Oncol 1990; 8: 994-1004.

[6] Dekker AW, Van't Veer MB, Sizoo W, et al. Intensive postremission chemotherapy without maintenance therapy in adult acute lymphoblastic leukemia. J Clin Oncol 1997; 14: 476-82.

[7] Offidani M, Corvatta L, Malerba L, et al. Comparsion of two regimens for the treatment of elderly patients with adult acute lymphoblastic leukemia(ALL). Leuk Lymph 2005; 46: 223-38.

[8] Blum KA, Lozanski G, Byrd JC. Adult Burkitt leukemia and lymphoma. Blood 2004; 104: 3009-20.

[9] Thiebaut A, Vernant JP, Degos L, et al. Adult acute lymphocytic leukemia study testing chemotherapy and autologous and allogeneic transplantation: a follow-up report of the French protocol LALA 87. Hematol Oncol Clin North Am 2000; 14: 1353-65.

[10] Larson RA. Recent clinical trials in acute lymphocytic leukemia by the Cancer and Leukemia Group B. Hematol Oncol Clin North Am 2000; 14: 1367-79.

[11] Garcia-Manero G, Kantarjian H. The Hyper-CVAD regimen in adult acute lymphocytic leukemia. Hematol Oncol Clin North Am 2000; 14: 1381-96.

[12] Larson RA, Dodge RK, Burns CP, et al. A five-drug remission induction regimen with intensive consolidation for adults with acute lymphoblastic leukemia: Cancer and Leukemia Group B Study 8811. Blood 1995; 85: 2025-37.

[13] Hussein KK, Dahlberg S, Head D, et al., and the Southwest Oncology Group. Treatment of acute lymphoblastic leukemia in adults with intensive induction, consolidation and maintenance chemotherapy. Blood 1989; 73: 57-63.

[14] Proctor SJ. Acute lymphoblastic leukemia in adults: the case for a strategic shift in study approach. Br J Haematol 1994; 88: 229-33.

[15] Arlin ZA, Feldman EJ, Finger LR, et al. Short course high dose mitoxantrone with high dose cytarabine is effective therapy for adult lymphoblastic leukemia. Leukemia 1991; 5: 712-14.

[16] Weiss, M, Maslak P, Feldman E, et al. With high-dose mitoxantrone induces rapid complete remissions in adult acute lymphoblastic leukemia without the use of vincristine or prednisone. J Clin Oncol 1994; 14: 2480-5.

[17] Kantarjian HM, O'Brien S, Smith TL, et al. Results of treatment with hyper-CVAD, a dose-intensive regimen, in adult acute lymphocytic leukemia. J Clin Oncol 2000; 18: 547-61.

[18] Kantarjian H, Thomas D, O'Brien S, et al. Long-term follow-up results of hyperfractionated cyclophosphamide, vincristine, doxorubicin, and dexamethasone (Hyper-CVAD), a dose-intensive regimen, in adult acute lymphocytic leukemia. Cancer 2004; 101: 2788-801.

[19] Radford JE Jr, Bums CP, Jones MP, et al. Adult acute lymphoblastic leukemia: Results of the Iowa HOP-L protocol. J Clin Oncol 1989; 7: 58-66.

[20] Ellison RR, Mick R, Cuttner J, et al. The effects of postinduction intensification treatment with cytarabine and daunorubicin in adult acute lymphocytic leukemia: A prospective randomized clinical trial by Cancer and Leukemia Group B. J Clin Oncol 1991; 9: 2002-15.

[21] Weiss MA, Aliff TB, Tallman MS, et al. High dose of idarubicin combined with cytarbine as induction therapy for adult patients with recurrent or refractory acute lymphoblastic leukemia. Cancer 2002; 95: 581-87. 
[22] Gaynor J, Chapman D, Little C, et al. A cause-specific hazard rate analysis of prognostic factors among 199 adults with acute lymphoblastic leukemia: The Memorial Hospital experience since 1969. J Clin Oncol 1988; 6: 1014-30.

[23] Hoelzer D, Thiel E, Loffier H, et al. Prognostic factors in a multicenter study for treatment of acute lymphoblastic leukemia in adults. Blood 1988; 71: 123-31.

[24] Morra E, Lazzarino M, Inverdadi D, et al. Systemic high-dose Ara$\mathrm{C}$ for the treatment of meningeal leukemia in adult acute lymphoblastic leukemia. And non-Hodgkin's lymphoma. J Clin Oncol 1986; 4: 1207-11.

[25] Ludwig W, Rieder H, Bartram C, et al. Immunophenotypic and genotypic features, clinical characteristics and treatment outcome of adult Pro-B acute lymphoblastic leukemia: results of the German multicenter trials GMALL 03/87 and 04/89. Blood 1998; 92: 1898909.

[26] Thomas DA, Faderl S, Cortes J, et al. Treatment of Philadelphia chromosome-positive acute lymphocytic leukemia with hyperCVAD and imatinib mesylate. Blood 2004; 103: 4396-407.

[27] Thomas D, O'Brien S, Cortes J, et al. Outcome with the hyperCVAD regimens in lymphoblastic lymphoma. Blood 2004; 104: 1624-30.
[28] Khouri IF, Romaguera J, Kantarjian H, et al. Hyper-CVAD and high dose methotrexate /cytarabine followed by stem cell transplantation: an active regimen for aggressive mantle cell lymphoma. J Clin Oncol 1998; 16: 3803-9.

[29] Dimopoulos MA, Weber D, Kantarjian H, et al. HyperCVAD for VAD-resistant multiple myeloma. Am J Hematol 1996; 52: 77-81.

[30] Thomas, D, Faderl S, O'Brien S, et al. Chemoimmunotherapy with hyper-CVAD plus rituximab for the treatment of adult burkitt and burkitt-type lymphoma or acute lymphoblastic leukemia. Cancer 2006; 106: 1569-80.

[31] Kurtzberg J, Keating M, Moore JO, et al. 2-Amino-9-Barabinosyl6-methoxy-9H-guanine (GW506U; Compound 506U) is highly active in patients with T-cell malignancies: Results of a phase I trial in pediatric and adult patients with refractory hematological malignancies. Blood 1996; 88: 699a, (abstr).

[32] Tedeschi A, Montillo M, Strocchi E, et al. High dose idarubicin in combined with Ara-C in patients with relapsed or refractory acute lymphoblastic leukemia: a pharmacokinetic and clinical study. Cancer Chemother Pharmacol 2007; 59: 771-98.

(C) Lu et al.; Licensee Bentham Open.

This is an open access article licensed under the terms of the Creative Commons Attribution Non-Commercial License (http: //creativecommons.org/licenses/by$\mathrm{nc} / 3.0 /$ ) which permits unrestricted, non-commercial use, distribution and reproduction in any medium, provided the work is properly cited. 